\title{
Prediction Validation of Two Glycaemic Control Models in Critical Care
}

\author{
Ulrike Pielmeier, J. Geoffrey Chase, \\ Steen Andreassen, Birgitte Steenfeldt Nielsen, Pernille Haure, \\ Geoffrey M. Shaw
}

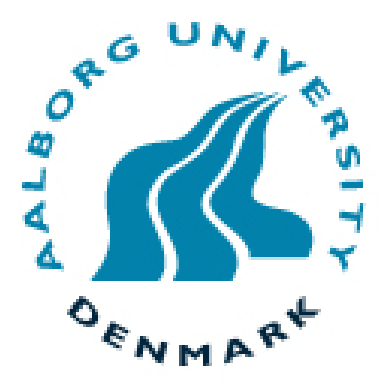

$\mathrm{UCF}$ 


\section{Hyperglycaemia in the ICU}

- Dysfunctional glucose regulatory mechanisms, due to stress

- Prevalent in critical care (10-65\%) [Krinstey, 2003; Umpierrez 2003]

- A marker of severity of illness

- Associated with increased:

- Mortality

- Treatment recommendations vary

- Sepsis

- Myocardial infarction

- Polyneuropathy

- Multiple-organ failure 


\section{Hyperglycaemia in the ICU}

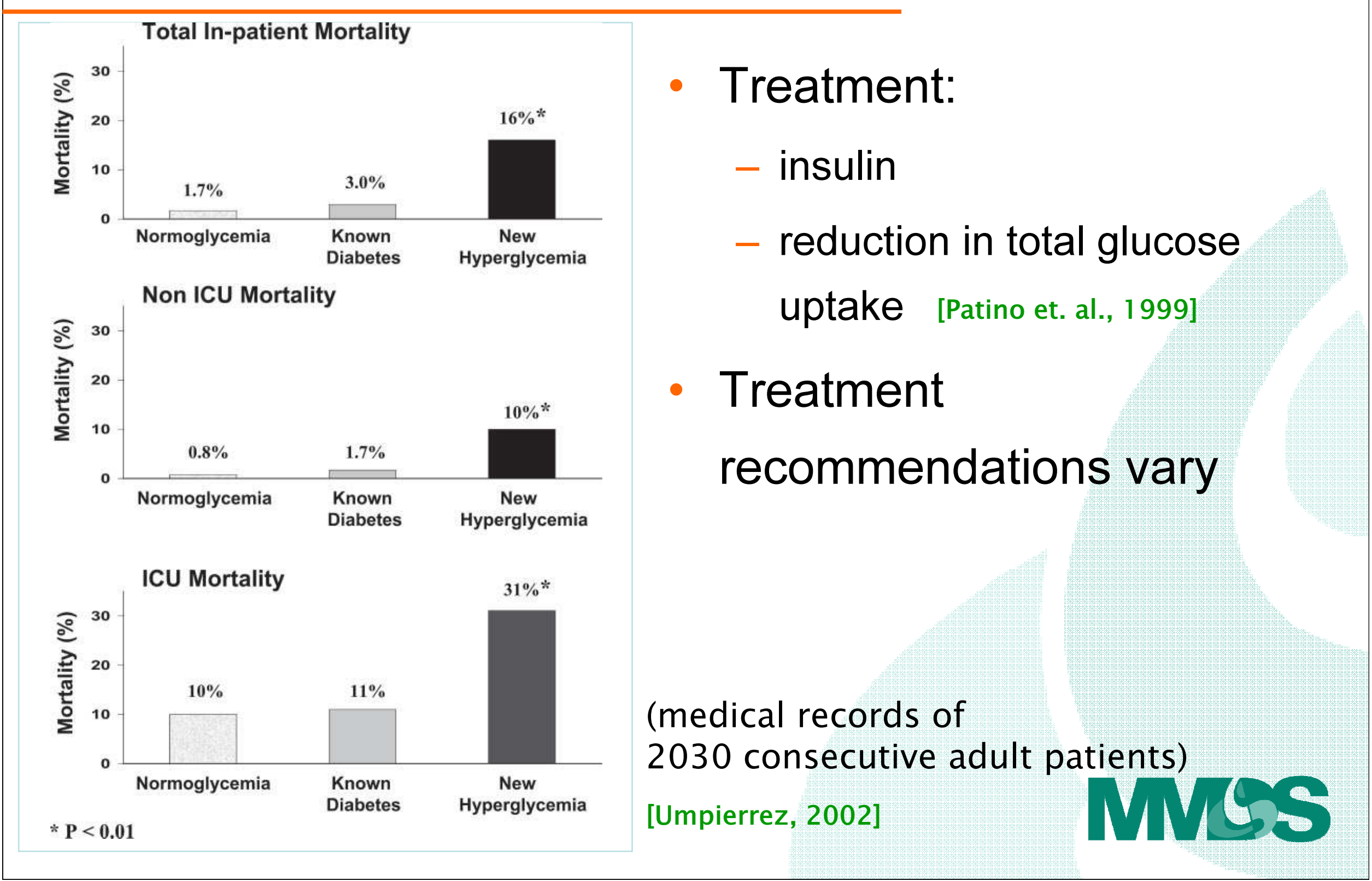




\section{Model-based blood glucose control}

- Predictive control to:

- Simulate outcomes of therapeutic interventions

- Help on scheduling of blood glucose measurements

- Give advice on insulin and/or nutrition

- Aim

- Ensure patient safety blood

- Facilitate treatment

- Reduce clinical burden

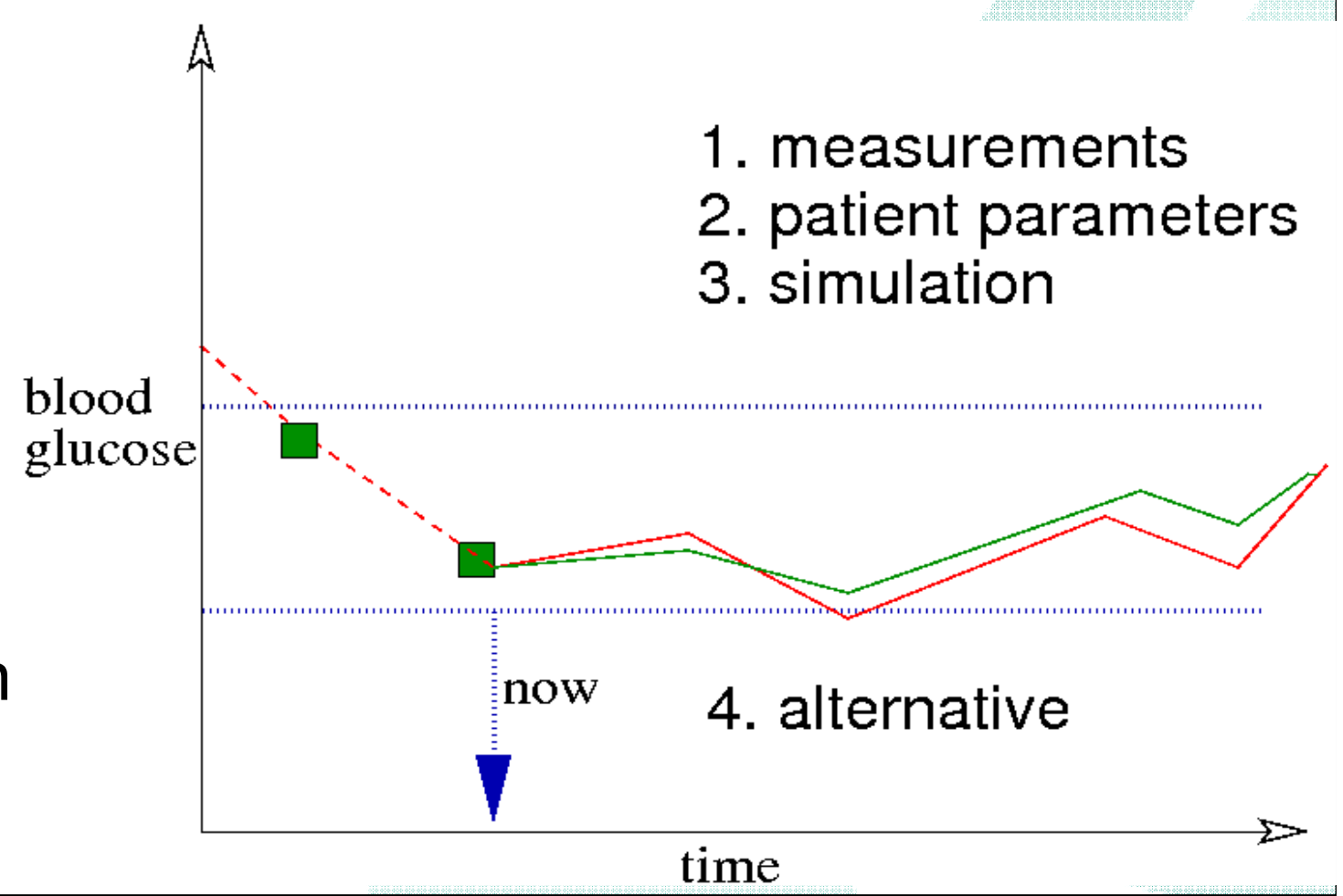




\section{The models}

\section{GlucoSafe model}

- Aalborg, DK

- Composite physiological model

- Based on work by Van Cauter et.al. (1992), Arleth et.al.(2000), Lotz et. al.(2005)

- Tested with retrospective patient data

- Clinical testing in preparation

\section{CC model}

- Christchurch, NZ

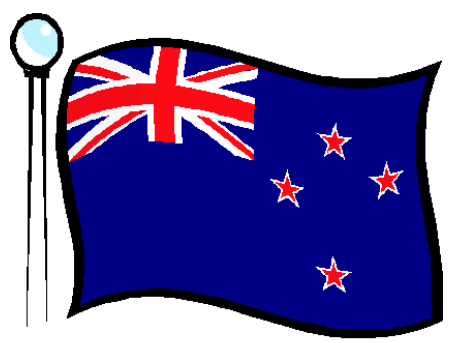

- Clinically validated (SPRINT + several trials)

- Good glycaemic control in 400+ general ICU patients:

- $54 \%$ measurements in the range 4.4-6.1 mmol//

- $0.02 \%<2.2 \mathrm{mmol} / \mathrm{l}$ ( $2 \%$ by patient)

- $35 \%$ reduction in hospital mortality $(\mathrm{P}=0.02)$

This study validates GlucoSafe using clinical data and in

\section{comparison to the CC model}




\section{The GlucoSafe model}

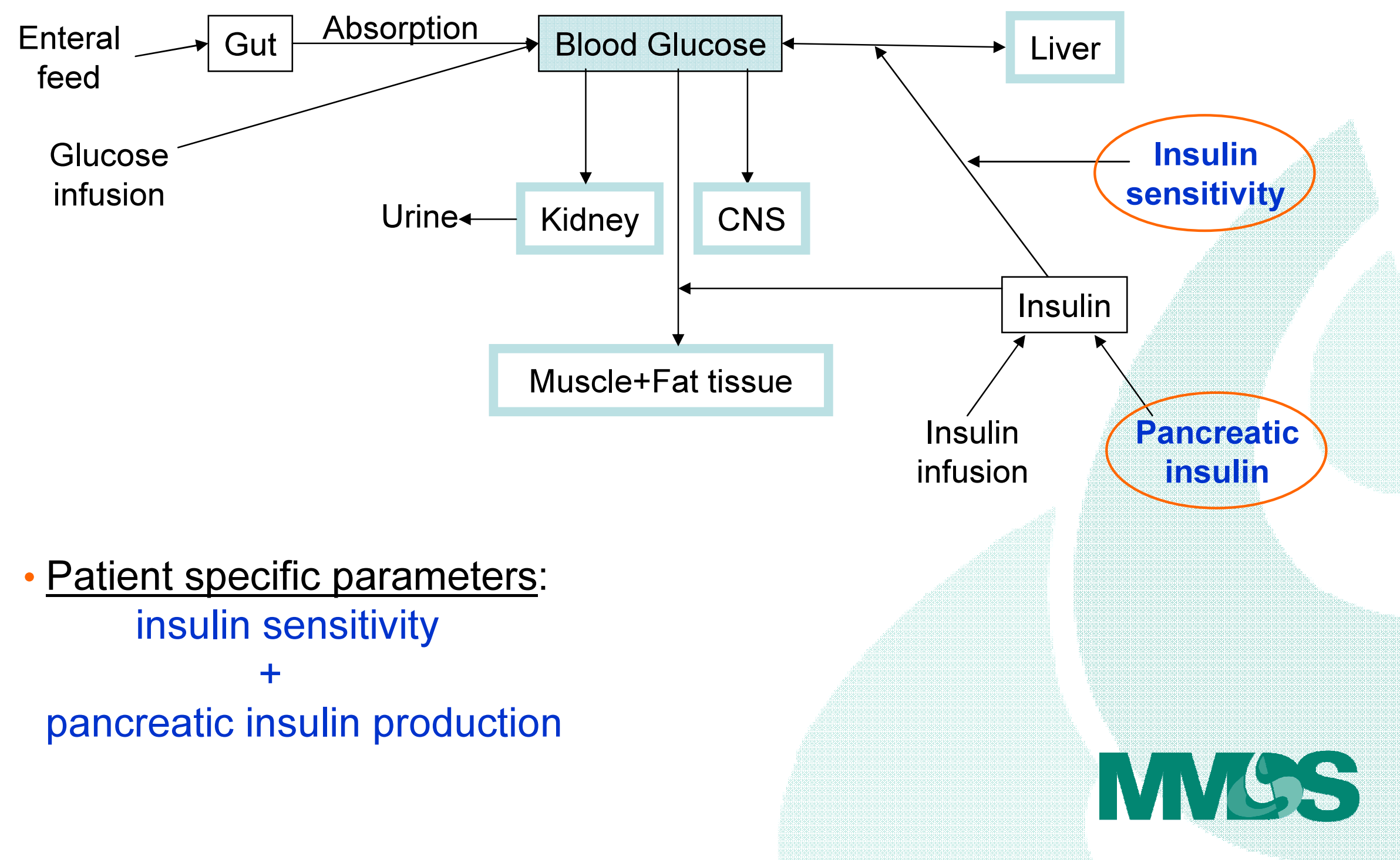




\section{The CC model (SPRINT protocol)}

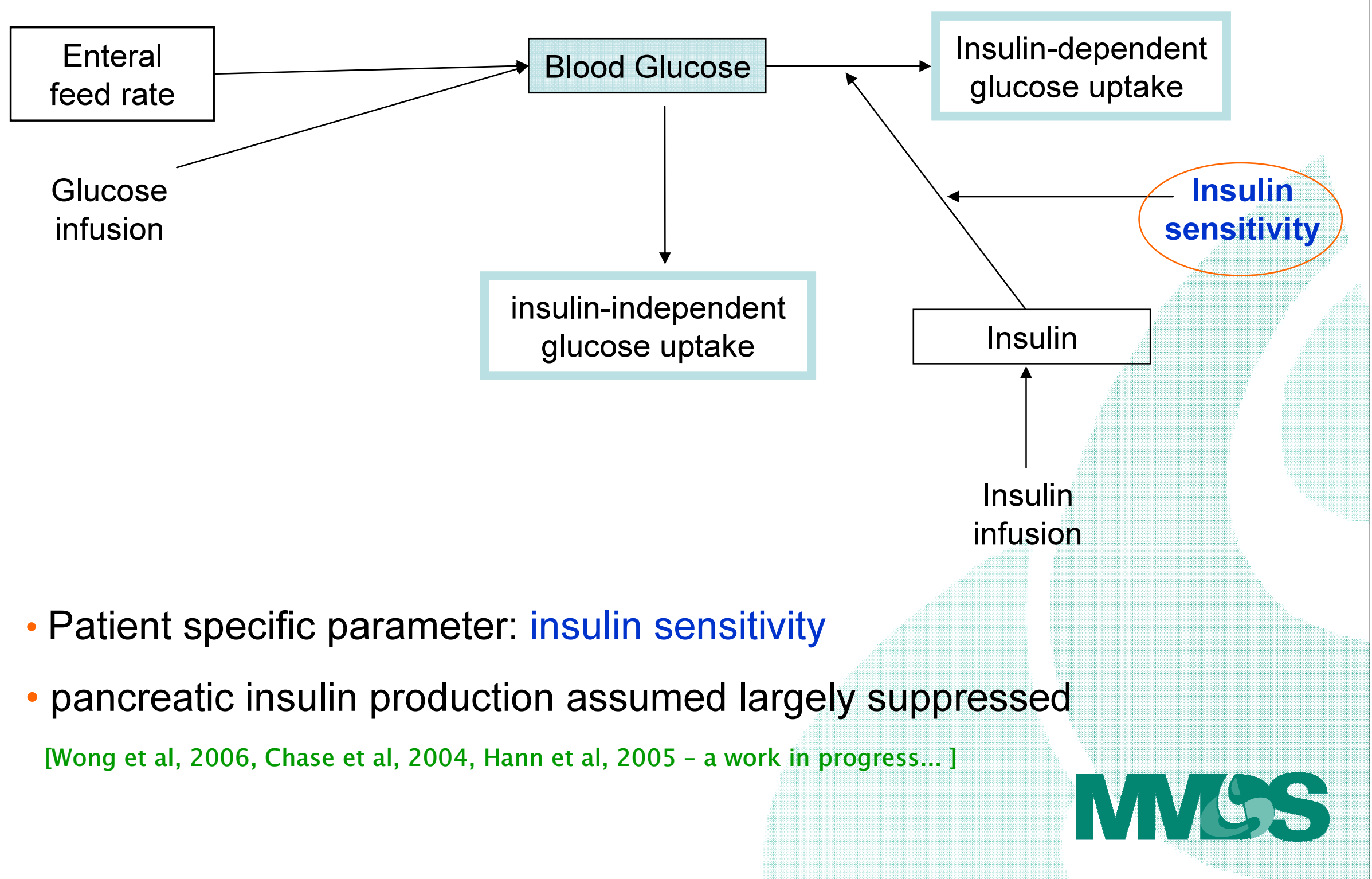




\section{Patient data}

- Retrospective data from 11 hyperglycaemic patients

- 5 trauma ICU patients (Aalborg, "DK" cohort)

- 6 medical ICU patients (Christchurch, "NZ" cohort pre-SPRINT)

- DK less critically ill than NZ

- Effectively 2 different cohorts

- Mean sampling interval:

- DK: $221 \mathrm{~min}$

- NZ: 154 min

- Mean \% (4-7 mmol/l):

- DK: $41 \%$

- NZ: $38 \%$

- 4 diabetic patients

- 2 type 2

- 2 type 1

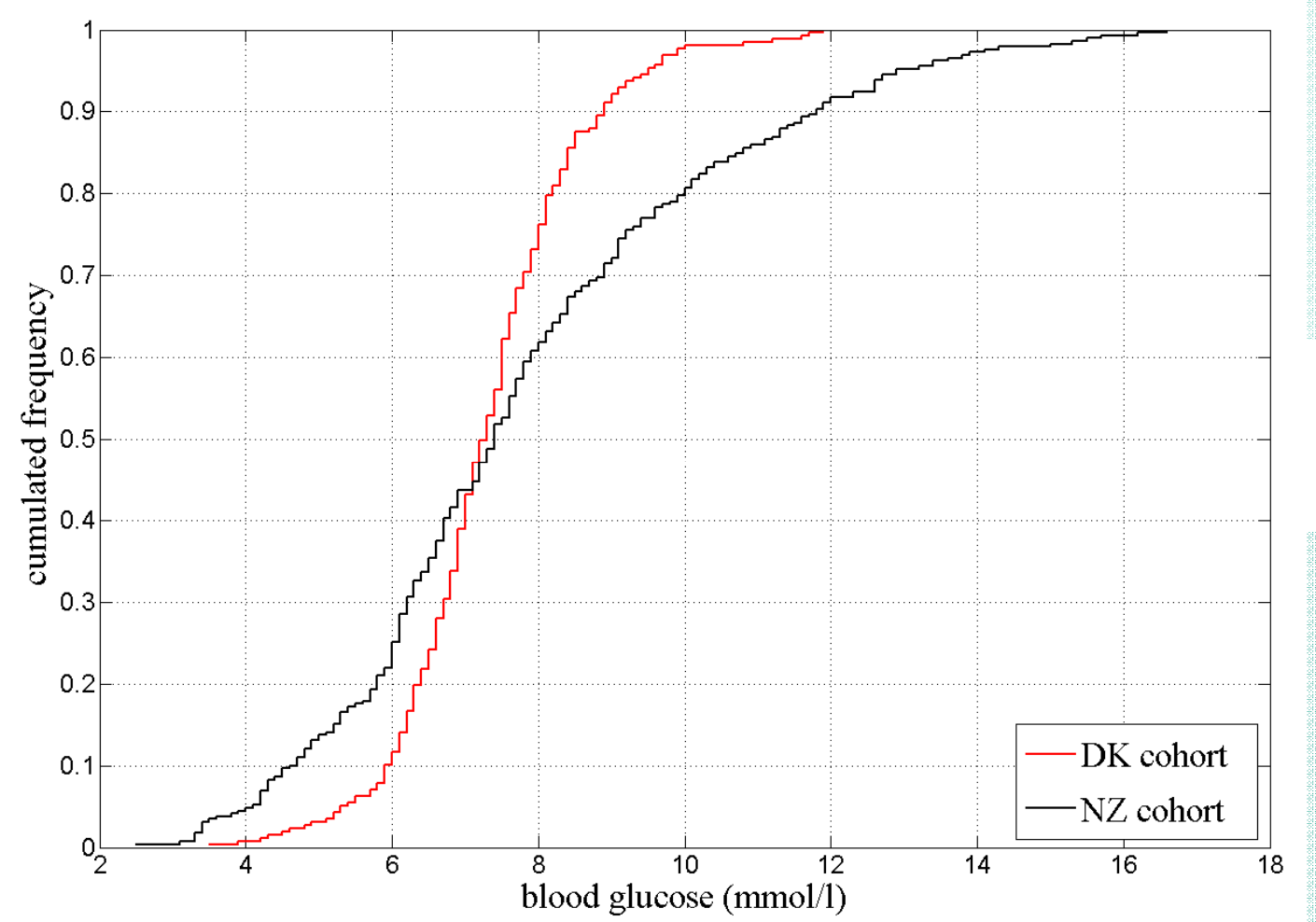




\section{Model prediction algorithm}

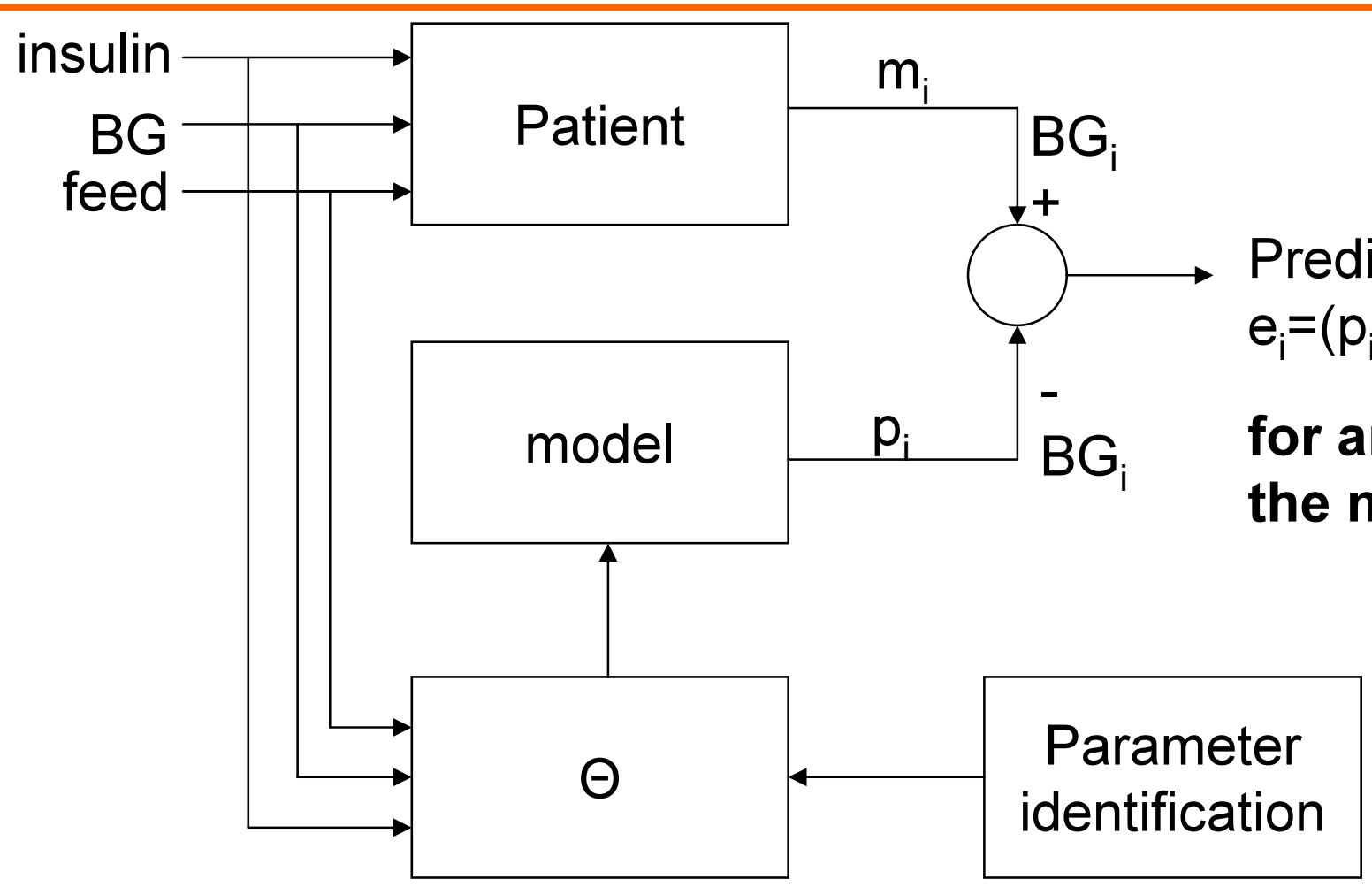

- Prediction errors "ordered" by hourly prediction interval

- Root mean square (RMS) calculated for each interval 


\section{RMS \% error prediction}

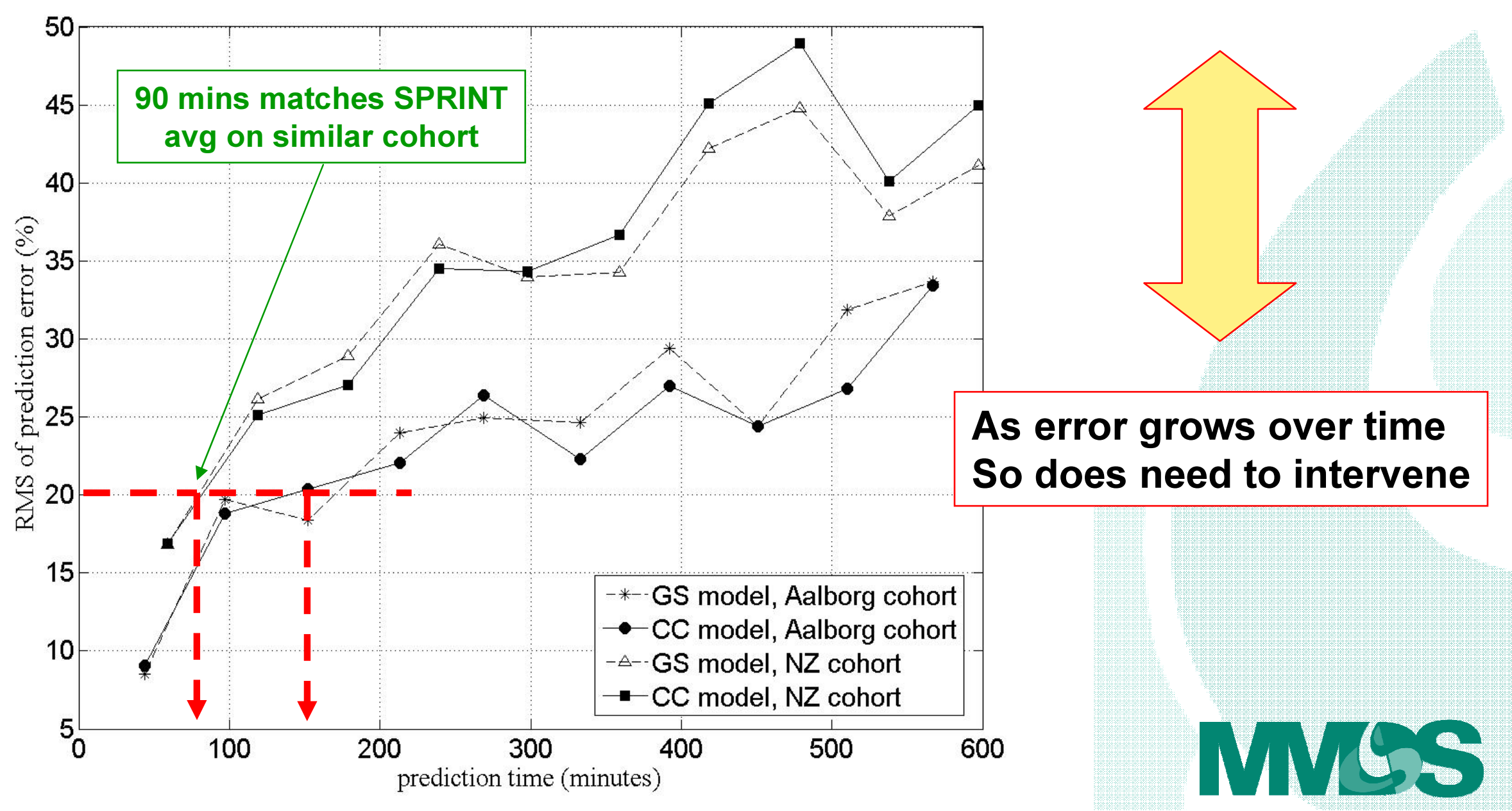




\section{RMS Prediction Error Summary}

- Median errors over all time periods can vary significantly by patient

$--5.4 \% \rightarrow 12.2 \%$ for GS

$--16.8 \rightarrow 9.7 \%$ for CC

- GS tends to overpredict with predominantly positive errors

- CC more even with some larger outliers extending range.

- Prediction errors are felt to be a better predictor of clinical utility than fitting errors as they represent or illustrate the model as it would be used 


\section{Conclusions}

- GlucoSafe is expected to be a safe and effective model for glycaemic control in intensive care

- Prediction accuracy and time to act depends on patient cohort (level of critical illness)

- The Future: advice, customization of models to cohort, influence of enteral glucose absorption, pancreatic secretion under insulin infusion... 


\section{Thank you for your attention}
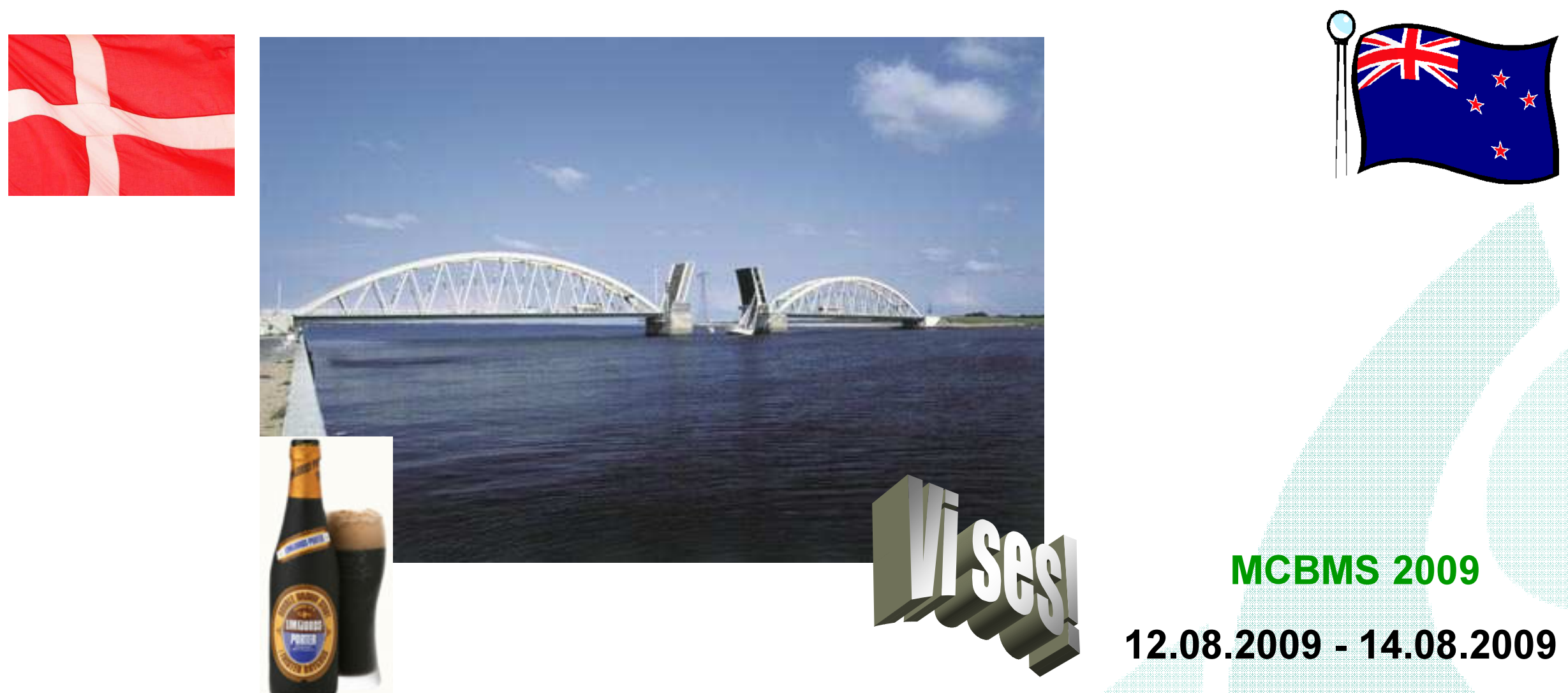

MCBMS 2009

12.08.2009 - 14.08.2009

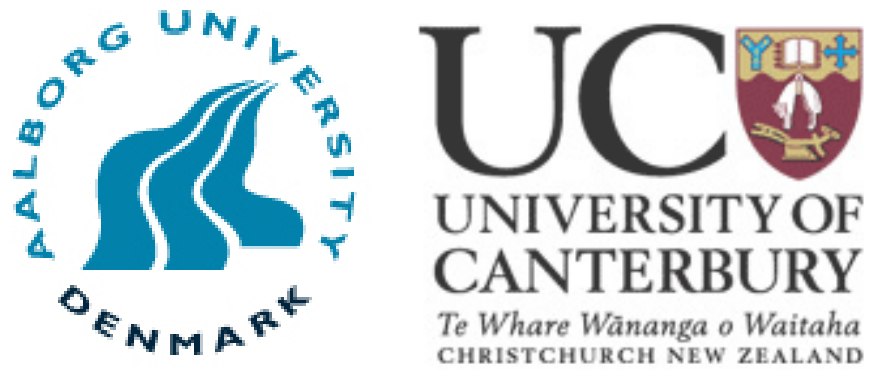




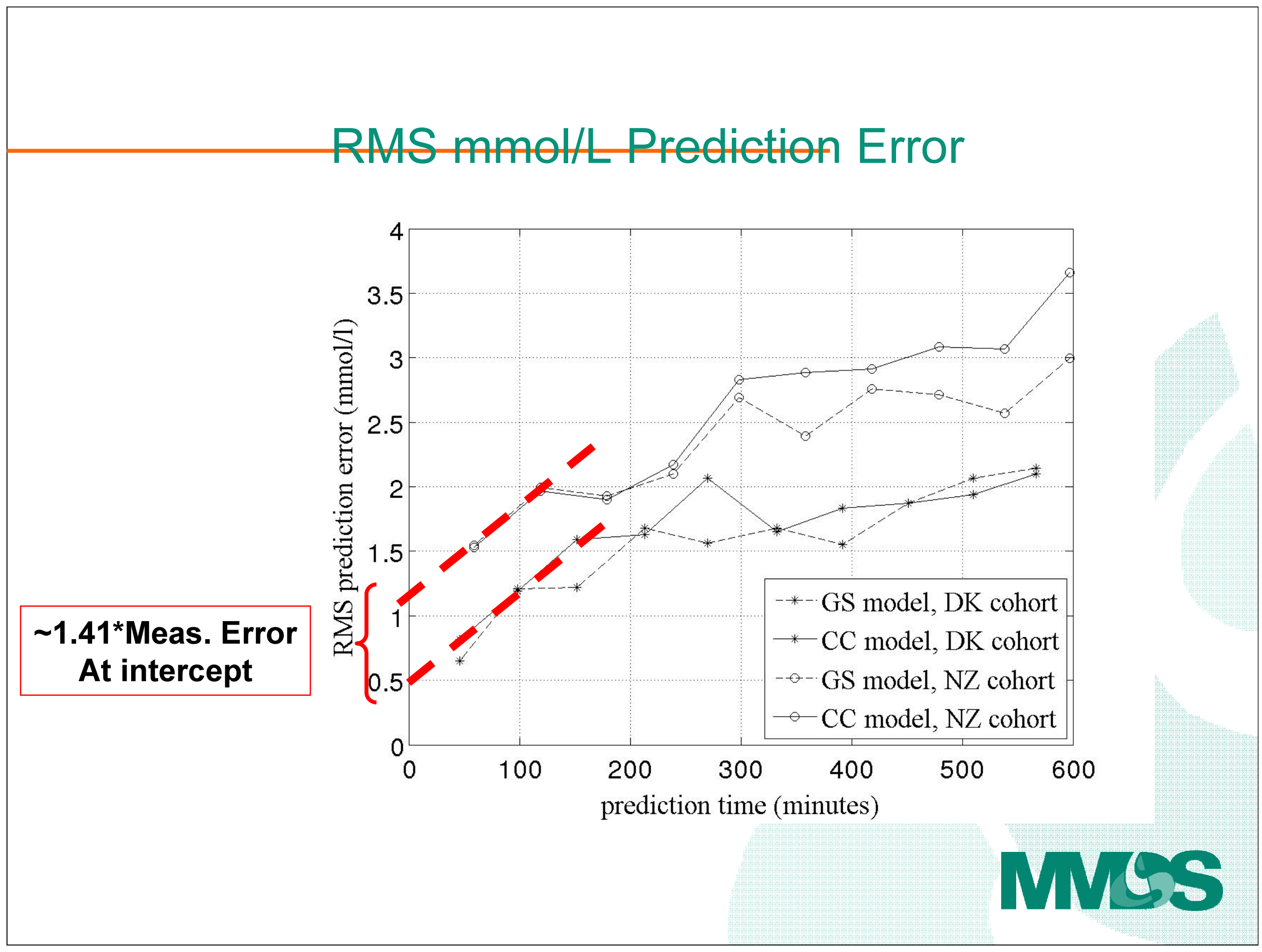




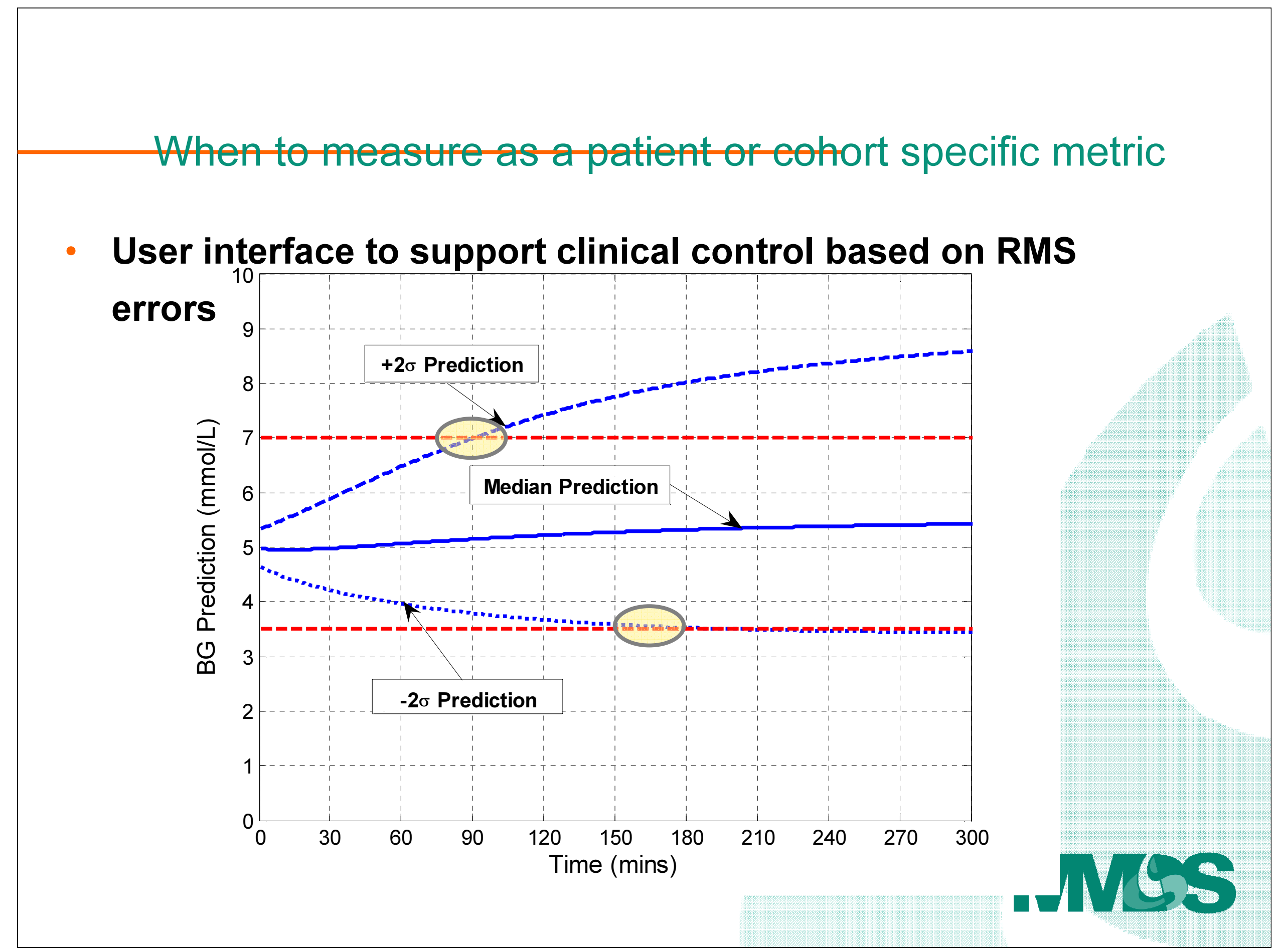

